JYFL-6/01

hep-ph/0104124

April 2001

\title{
OBTAINING THE NUCLEAR GLUON DISTRIBUTION FROM HEAVY QUARK DECAYS TO LEPTON PAIRS IN $\mathrm{p} A$ COLLISIONS
}

\author{
K.J. Eskola ${ }^{\mathrm{a}, \mathrm{b}}$ ], V.J. Kolhinen ${ }^{\mathrm{a}}$ ? and R. Vogte \\ ${ }^{a}$ Department of Physics, University of Jyväskylä, \\ P.O. Box 35, FIN-40351 Jyväskylä, Finland \\ ${ }^{\mathrm{b}}$ Helsinki Institute of Physics, \\ P.O. Box 64, FIN-00014 University of Helsinki, Finland \\ ${ }^{\mathrm{c}}$ Lawrence Berkeley National Laboratory, Berkeley, CA 94720, USA, \\ and \\ Physics Department, University of California, Davis, CA 95616, USA
}

\begin{abstract}
We have studied how lepton pairs from decays of heavy-flavoured mesons produced in $\mathrm{p} A$ collisions can be used to determine the modifications of the gluon distribution in the nucleus. Since heavy quark production is dominated by the $g g$ channel, the ratio of correlated lepton pair cross sections from $D \bar{D}$ and $B \bar{B}$ decays in $\mathrm{p} A$ and pp collisions directly reflects the ratio $R_{g}^{A} \equiv f_{g}^{A} / f_{g}^{p}$. We have numerically calculated the lepton pair cross sections from these decays in pp and $\mathrm{p} A$ collisions at SPS, RHIC and LHC energies. We find that ratio of the $\mathrm{p} A$ to $\mathrm{pp}$ cross sections agrees quite well with the input $R_{g}^{A}$. Thus, sufficiently accurate measurements could be used to determine the nuclear modification of the gluon distribution over a greater range of $x$ and $Q^{2}$ than presently available, putting strong constraints on models.
\end{abstract}

\footnotetext{
${ }^{1}$ kari.eskola@phys.jyu.fi

${ }^{2}$ vesa.kolhinen@phys.jyu.fi

${ }^{3}$ vogt@lbl.gov
} 


\section{Introduction}

Inclusive differential cross sections of hard scatterings in proton-nucleus collisions at high energies are, to first approximation, computable assuming factorization. For processes not sensitive to isospin effects, such as heavy quark production, these cross sections can be expressed as

$$
d \sigma\left(Q^{2}, \sqrt{s}\right)_{\mathrm{p} A \rightarrow a+X}=\sum_{i, j=q, \bar{q}, g} f_{i}^{\mathrm{p}}\left(x_{1}, Q^{2}\right) \otimes A f_{j}^{A}\left(x_{2}, Q^{2}\right) \otimes d \hat{\sigma}\left(Q^{2}, x_{1}, x_{2}\right)_{i j \rightarrow a+x}
$$

where $\hat{\sigma}\left(Q^{2}, x_{1}, x_{2}\right)_{i j \rightarrow a+x}$ are the perturbatively calculable partonic cross sections for producing parton $a$ at scale $Q^{2} \gg \Lambda_{\mathrm{QCD}}^{2}, x_{1}$ and $x_{2}$ are the momentum fractions of the partons involved in the hard scattering, while $f_{i}^{\mathrm{p}}$ and $f_{i}^{A}$ are the distributions of parton $i$ in a free proton and a nucleus with mass number $A$ respectively. In the high- $Q^{2}$ limit, possible multiple scatterings of individual partons [1], 2] are neglected but the parton distributions will be modified in the nucleus [3, 4. Equation (11) may be generalized to nucleus-nucleus collisions but we focus only on $p A$ collisions in this paper.

The measurements of the structure function $F_{2}^{A}$ in deep inelastic lepton-nucleus scattering (DIS) clearly show that, compared to deuterium, D, the ratios $R_{F_{2}}^{A} \equiv$ $F_{2}^{A} / F_{2}^{\mathrm{D}}$, differ from unity. As nuclear effects in $\mathrm{D}$ are negligible, this indicates that the parton distributions of bound nucleons are different from those of a free proton, $R_{i}^{A}\left(x, Q^{2}\right) \equiv f_{i}^{A}\left(x, Q^{2}\right) / f_{i}^{\mathrm{p}}\left(x, Q^{2}\right) \neq 1$. The nuclear effects observed in $F_{2}^{A}$ are usually categorized according to the different regions of Bjorken- $x$ as: shadowing, $R_{F_{2}}^{A} \leq 1$, at $x \lesssim 0.1$; antishadowing, $R_{F_{2}}^{A} \geq 1$, at $0.1 \lesssim x \lesssim 0.3$; the EMC effect, $R_{F_{2}}^{A} \leq 1$, at $0.3 \lesssim x \lesssim 0.7$; and Fermi motion, $R_{F_{2}}^{A} \geq 1$, at $x \rightarrow 1$ and beyond. For a review of the measurements and some models of nuclear effects, see Ref. [5].

The scale evolution of the nuclear parton distributions is, to a first approximation, similar to that of the free proton. Thus at scales $Q^{2}>Q_{0}^{2} \gg \Lambda_{\mathrm{QCD}}^{2}$ the evolution of $f_{i}^{A}\left(x, Q^{2}\right)$ can be described by the Dokshitzer-Gribov-Lipatov-Altarelli-Parisi (DGLAP) evolution equations [ [6] if the initial conditions are given at a fixed initial scale $Q_{0}^{2}$. Such a DGLAP analysis of the nuclear parton densities [3, 4, 7] assumes that the nuclear effects factorize from the parton densities for $Q_{0}^{2} \gtrsim 1 \mathrm{GeV}^{2}$ and the subsequent $Q^{2}$-evolution of the modifications follows the DGLAP equations, neglecting $\sim 1 / Q^{2}$ corrections $\left[8\right.$, 9]. The nuclear modifications at $Q_{0}^{2}, R_{i}^{A}\left(x, Q_{0}^{2}\right)$, can then be determined based on constraints provided by the measurements of $F_{2}^{A}$ in DIS, the Drell-Yan cross sections in $p A$ collisions [10, 11] and conservation of momentum and baryon number. This procedure was carried out in Ref. [3]. In the present work, we utilize the EKS98 parametrization of the nuclear effects $R_{i}^{A}\left(x, Q^{2}\right)$, obtained from Ref. [3], released for public use in Ref. [4].

There remain uncertainties, especially in the determination of the nuclear gluon distributions. Figure 1 shows the gluon modifications, $R_{g}^{A}\left(x, Q^{2}\right)$, for a heavy nucleus, $A=208$, as obtained from a DGLAP analysis [3, \#. So far there are no direct 


\section{$A=208$}

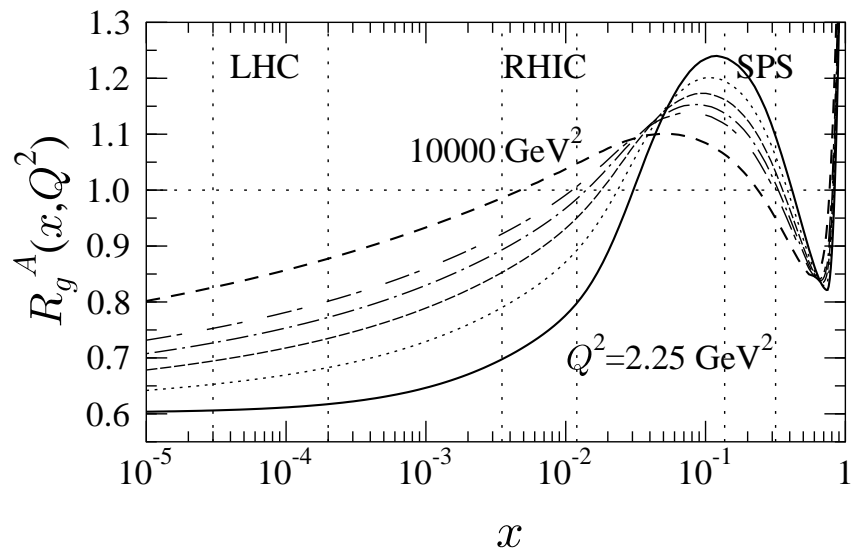

Figure 1: Scale evolution of the ratio $R_{g}^{A}\left(x, Q^{2}\right)$ for an isoscalar nucleus $A=208$ according to EKS98 [3, 国. The ratios are shown as functions of $x$ at fixed values of $Q^{2}$ equidistant in $\log Q^{2}: 2.25 \mathrm{GeV}^{2}$ (solid), $5.39 \mathrm{GeV}^{2}$ (dotted), $14.7 \mathrm{GeV}^{2}$ (dashed), $39.9 \mathrm{GeV}^{2}$ (dotteddashed), $108 \mathrm{GeV}^{2}$ (double-dashed), and $10000 \mathrm{GeV}^{2}$ (dashed). The regions between the vertical dashed lines show the dominant values of $x_{2}$ probed by muon pair production from $D \bar{D}$ at SPS, RHIC and LHC energies.

constraints on the nuclear gluon distributions. These can, however, be constrained indirectly through the scale evolution of $F_{2}^{A}\left(x, Q^{2}\right)$ [3], 13. The only measured $Q^{2}$ dependence is of the ratio $F_{2}^{\mathrm{Sn}} / F_{2}^{\mathrm{C}}[\mathbb{1 2}$ at $0.02<x<0.2$. At other values of $x$, only the requirement of stability of the evolution can be used in the perturbative analysis. Finally, a global limit on the nuclear gluon distribution is provided by momentum conservation which effectively determines the level of antishadowing in $R_{g}^{A}\left(x, Q_{0}^{2}\right)$. More measurement-based constraints are thus vital for reducing the uncertainties in the nuclear gluon distributions. Heavy quark production is one such process.

The dominant subprocess of heavy quark production in hadronic or nuclear collisions is $g g \rightarrow Q \bar{Q}$. The produced heavy quarks fragment into mesons which may decay semi-leptonically. Lepton pairs formed from these decay leptons thus carry direct information about the input nuclear gluon distribution. Previous studies can be found in Refs. [14, 15]. At that time, however, $R_{g}^{A}$ was not yet as well constrained as in the EKS98 parameterization.

There are clear advantages for studying the nuclear gluon distribution in $\mathrm{p} A$ interactions. In $\mathrm{p} A$ studies, any possible complications of dense collective matter produced in ultrarelativistic $A A$ collisions are avoided. Also, the number of uncorrelated pairs where e.g. the $D$ and $\bar{D}$ originate in two independent partonic subcollisions remains rather small. 
The purpose of this paper is to study to what extent lepton pair production from heavy flavor meson decays reflects nuclear effects on the gluon distributions in $\mathrm{p} A$ collisions, especially in the ratio $\sigma^{\mathrm{p} A} / \sigma^{\mathrm{pp}}$. As the input nuclear gluon modification, we will use the EKS98 gluon parameterization shown in Fig. 1. Both correlated lepton pairs, where the $Q \bar{Q}$ pair is created in one subcollision, and uncorrelated pairs are considered. To probe the ratio $R_{g}^{A}\left(x, Q^{2}\right)$ over as wide an $x$ range as possible, we study $\mathrm{p} A$ and pp collisions at the SPS $(\sqrt{s}=17.3 \mathrm{GeV})$, RHIC $(\sqrt{s}=200 \mathrm{GeV})$ and LHC $(\sqrt{s}=5500 \mathrm{GeV})$ assuming the same energy as in $A A$ collisions for both pp and $\mathrm{p} A$ studies. Lepton pair production from $D \bar{D}$ decays at the SPS (NA60) will probe the antishadowing region while at RHIC (PHENIX, STAR) and LHC (ALICE, CMS) the cross sections are predominantly sensitive to the gluon properties in the shadowing region, as indicated in Fig. [1].

It should be noted that the present work focuses only on the best-case scenario in which we assume that the leptons from heavy meson decays can be identified through vertex displacement. We make no attempt to simulate the background processes in detail here. This study, together with Refs. [14, 15], should provide further motivation for performing more detailed event simulations in real detectors. Our hope is that the lepton pair cross sections measured in the future could be used to constrain the remaining uncertainties in the nuclear gluon distribution in a model-independent way.

\section{Formalism}

\subsection{Lepton pair production in $\mathrm{p} A$ collisions}

We first present the expression for the differential cross section of lepton pair production in $\mathrm{p} A$ collisions. To simplify notation, we refer to generic heavy quarks, $Q$, and heavyflavoured mesons, $H$. In general, the lepton pair production cross section is

$$
\begin{aligned}
\frac{d \sigma^{\mathrm{p} A \rightarrow \bar{l}+X}}{d M_{\bar{l}} d y_{\bar{l}}}= & \int d^{3} \vec{p}_{l} d^{3} \vec{p}_{\bar{l}} \int d^{3} \vec{p}_{H} d^{3} \vec{p}_{\bar{H}} \delta\left(M_{\bar{l}}-M\left(p_{l}, p_{\bar{l}}\right)\right) \delta\left(y_{\bar{l}}-y\left(p_{l}, p_{\bar{l}}\right)\right) \\
& \times \frac{d \Gamma^{H \rightarrow l+X}\left(\vec{p}_{H}\right)}{d^{3} \vec{p}_{l}} \frac{d \Gamma^{\bar{H} \rightarrow \bar{l}+X}\left(\vec{p}_{\bar{H}}\right)}{d^{3} \vec{p}_{\bar{l}}} \frac{d \sigma^{\mathrm{p} A \rightarrow H \bar{H}+X}}{d^{3} \vec{p}_{H} d^{3} \vec{p}_{\bar{H}}} \\
& \times \theta\left(y_{\min }<y_{l}, y_{\bar{l}}<y_{\max }\right) \theta\left(\phi_{\min }<\phi_{l}, \phi_{\bar{l}}<\phi_{\max }\right) .
\end{aligned}
$$

where $M_{\overline{l l}}$ and $y_{\bar{l}}$ are the mass and the rapidity of the lepton pair, defined as

$$
\begin{aligned}
M\left(p_{l}, p_{\bar{l}}\right) & =\sqrt{\left(p_{l}+p_{\bar{l}}\right)^{2}} \\
y\left(p_{l}, p_{\bar{l}}\right) & =\frac{1}{2} \ln \left(\frac{\left(E_{l}+E_{\bar{l}}\right)+\left(p_{l z}+p_{\bar{l} z}\right)}{\left(E_{l}+E_{\bar{l}}\right)-\left(p_{l z}+p_{\overline{l z}}\right)}\right) .
\end{aligned}
$$


The decay rate, $d \Gamma^{H \rightarrow l+X}\left(\vec{p}_{H}\right) / d^{3} \vec{p}_{l}$, is the probability that meson $H$ with momentum $\vec{p}_{H}$ decays to a lepton $l$ with momentum $\vec{p}_{l}$. The $\theta$ functions define single lepton rapidity and azimuthal angle cuts used to simulate detector acceptances.

Using a fragmentation function $D_{Q}^{H}$ to describe quark fragmentation to mesons, the $H \bar{H}$ production cross section can be written as

$$
\begin{aligned}
\frac{d \sigma^{\mathrm{p} A \rightarrow H \bar{H}+X}}{d^{3} \vec{p}_{H} d^{3} \vec{p}_{\bar{H}}} & =\int \frac{d^{3} \vec{p}_{Q}}{E_{Q}} \frac{d^{3} \vec{p}_{\bar{Q}}}{E_{\bar{Q}}} E_{Q} E_{\bar{Q}} \frac{d \sigma^{\mathrm{p} A \rightarrow Q \bar{Q}+X}}{d^{3} \vec{p}_{Q} d^{3} \vec{p}_{\bar{Q}}} \int_{0}^{1} d z_{1} D_{Q}^{H}\left(z_{1}\right) \int_{0}^{1} d z_{2} D \overline{\bar{Q}}\left(z_{2}\right) \\
& \times \delta^{(3)}\left(\vec{p}_{H}-z_{1} \vec{p}_{Q}\right) \delta^{(3)}\left(\vec{p}_{\bar{H}}-z_{2} \vec{p}_{\bar{Q}}\right)
\end{aligned}
$$

where $z$ is the fraction of the parent quark momentum carried by the final-state meson.

Quark fragmentation has been described by several different models. A fragmentation function smears the quark momentum into the momentum of the final-state meson during hadronization. Typical fragmentation functions are a delta function or the Peterson function [16]. In this study we have used the delta function but have also checked our results using the Peterson function. Delta function fragmentation simply assumes that the quark and meson momenta are identical,

$$
D_{Q}^{H}(z)=\delta(1-z)
$$

On the other hand, the Peterson function assumes that the meson momentum is smeared according to

$$
D_{Q}^{H}(z)=\frac{N}{z\left(1-(1 / z)-\epsilon_{Q} /(1-z)\right)^{2}}
$$

The normalization, $N$, is fixed by the requirement $\sum_{H} \int_{0}^{1} d z D_{Q}^{H}(z)=1$. The peak of the fragmentation function is at $z \approx 1-2 \epsilon_{Q}$ with a width $\epsilon_{Q} \approx\left(m_{q} / m_{Q}\right)^{2}$ [16]. We have assumed e.g. that all the $c$ quarks fragment into $D$ mesons so that $H \equiv D$ for charm.

The hadronic heavy quark production cross section per nucleon in $\mathrm{p} A$ collisions can be factorized into the general form

$$
\frac{1}{A} E_{Q} E_{\bar{Q}} \frac{d \sigma^{\mathrm{p} A \rightarrow Q \bar{Q}+X}}{d^{3} \vec{p}_{Q} d^{3} \vec{p}_{\bar{Q}}}=\sum_{i, j} \int_{0}^{1} d x_{1} \int_{0}^{1} d x_{2} f_{i}^{\mathrm{p}}\left(x_{1}, Q^{2}\right) f_{j}^{A}\left(x_{2}, Q^{2}\right) E_{Q} E_{\bar{Q}} \frac{d \hat{\sigma}^{i j \rightarrow Q \bar{Q}}}{d^{3} \vec{p}_{Q} d^{3} \vec{p}_{\bar{Q}}}
$$

The parton distributions $f_{i}^{\mathrm{p}}$ and $f_{j}^{A}$, described in Eq. (1), are evaluated at the scale $Q^{2} \sim m_{T_{Q}}^{2}$. To lowest order, LO, the partonic cross section is

$$
E_{Q} E_{\bar{Q}} \frac{d \hat{\sigma}^{i j \rightarrow Q \bar{Q}}}{d^{3} \vec{p}_{Q} d^{3} \vec{p}_{\bar{Q}}}=\frac{\hat{s}}{2 \pi} \frac{d \hat{\sigma}^{i j \rightarrow Q \bar{Q}}}{d \hat{t}} \delta^{(4)}\left(p_{1}+p_{2}-p_{Q}-p_{\bar{Q}}\right)
$$




\begin{tabular}{cccc}
\hline \hline & SPS $(A=208)[\mu \mathrm{b}]$ & $\operatorname{RHIC}(A=197)[\mu \mathrm{b}]$ & $\operatorname{LHC}(A=208)[\mu \mathrm{b}]$ \\
\hline$\tilde{\sigma}_{\mathrm{pp}}^{c \bar{c}}$ & 1.188 & 143.7 & 6137 \\
$\tilde{\sigma}_{\mathrm{p} A}^{c \bar{c}}$ & 1.282 & 140.1 & 4826 \\
$\tilde{\sigma}_{\mathrm{pp}}^{b \bar{b}}$ & $1.674 \times 10^{-5}$ & 1.412 & 251.9 \\
$\tilde{\sigma}_{\mathrm{p} A}^{b \bar{b}}$ & $1.464 \times 10^{-5}$ & 1.513 & 226.7 \\
\hline \hline
\end{tabular}

Table 1: The total $Q \bar{Q}$ production cross sections per nucleon in $\mathrm{pp}$ and $\mathrm{p} A$ interactions.

where $p_{1}$ and $p_{2}$ are the four momenta of the incoming partons. The lowest order convolution of the parton distributions with the subprocess cross section is written as

$$
\begin{gathered}
\sum_{i, j} f_{i}^{\mathrm{p}}\left(x_{1}, Q^{2}\right) f_{j}^{A}\left(x_{2}, Q^{2}\right) \frac{d \hat{\sigma}^{i j \rightarrow Q \bar{Q}}}{d \hat{t}}=\frac{1}{16 \pi \hat{s}^{2}}\left(f_{g}^{\mathrm{p}}\left(x_{1}, Q^{2}\right) f_{g}^{A}\left(x_{2}, Q^{2}\right){\overline{\mid \mathcal{M}_{g g \rightarrow Q \bar{Q}}}}^{2}\right. \\
\left.+\sum_{q=u, d, s}\left[f_{q}^{\mathrm{p}}\left(x_{1}, Q^{2}\right) f_{\bar{q}}^{A}\left(x_{2}, Q^{2}\right)+f_{\bar{q}}^{\mathrm{p}}\left(x_{1}, Q^{2}\right) f_{q}^{A}\left(x_{2}, Q^{2}\right)\right]\left|\overline{\mathcal{M}}_{q \bar{q} \rightarrow Q \bar{Q}}\right|^{2}\right)
\end{gathered}
$$

The squared matrix elements ${\overline{\mid \mathcal{M}_{g g \rightarrow Q \bar{Q}}}}^{2}$ and ${\overline{\mid \mathcal{M}_{q \bar{q} \rightarrow Q \bar{Q}}}}^{2}$ can be found in e.g. Ref. [17].

Integration of Eqs. (2) and (5) over the total phase space gives the normalization $\sigma^{\mathrm{p} A \rightarrow l \bar{l}}=B^{2} \sigma^{\mathrm{p} A \rightarrow H \bar{H}}=B^{2} \sigma^{\mathrm{p} A \rightarrow Q \bar{Q}}$, where $B=\Gamma_{l} / \Gamma$. The total cross section for lepton pair production is then equal to the total cross section of heavy quark pair production multiplied by the square of the lepton branching ratio. The following expressions for the double differential distribution and the total cross section of $Q \bar{Q}$ production per nucleon will be useful for our later discussion:

$$
\begin{aligned}
\frac{d \tilde{\sigma}^{Q \bar{Q}}}{d^{3} \vec{p}_{Q} d^{3} \vec{p}_{\bar{Q}}} & =\frac{1}{E_{Q}} \frac{1}{E_{\bar{Q}}}\left[\frac{1}{A} E_{Q} E_{\bar{Q}} \frac{d \sigma^{\mathrm{p} A \rightarrow Q \bar{Q}+X}}{d^{3} \vec{p}_{Q} d^{3} \vec{p}_{\bar{Q}}}\right] \\
\tilde{\sigma}^{Q \bar{Q}} & =\int d^{3} \vec{p}_{Q} d^{3} \vec{p}_{\bar{Q}} \frac{d \tilde{\sigma}^{Q \bar{Q}}}{d^{3} \vec{p}_{Q} d^{3} \vec{p}_{\bar{Q}}} \\
& =\int d p_{T}^{2} d y_{1} d y_{2} \sum_{i j} x_{1} f_{i}^{p}\left(x_{1}, Q^{2}\right) x_{2} f_{j}^{A}\left(x_{2}, Q^{2}\right) \frac{d \hat{\sigma}^{i j \rightarrow Q \bar{Q}}}{d \hat{t}}
\end{aligned}
$$

where $x_{1,2}=m_{T} / \sqrt{s}\left(e^{ \pm y_{1}}+e^{ \pm y_{2}}\right)$ and $m_{T}^{2}=p_{T}^{2}+m_{Q}^{2}$. The rapidities of the heavy quarks are $y_{1,2}$. The total $Q \bar{Q}$ cross sections per nucleon in pp and $\mathrm{p} A$ interactions are presented in Table 1.

\subsection{Correlated and uncorrelated pairs in $\mathrm{p} A$ collisions}

In $\mathrm{p} A$ collisions, a number of uncorrelated lepton pairs are also produced along with the correlated lepton pairs. Since, in most cases, one would prefer to study either correlated 
or uncorrelated pairs alone, these events must be separated. One method of separation is like-sign subtraction because like-sign pairs are all uncorrelated: $l^{+}$'s originate from e.g. $c$ quarks whereas $l^{-}$'s come from $\bar{c}$ quarks. Another possible method is to use azimuthal angle cuts. At lowest order, correlated $Q \bar{Q}$ pairs are produced back-toback. Thus, leptons produced from these pairs tend to have a larger relative azimuthal angle than uncorrelated ones. With a suitable azimuthal cut the relative amount of uncorrelated pairs could possibly be reduced. However, the focus of our study is not how to separate the pairs but an estimate of their rates.

In order to calculate the number of correlated and uncorrelated pairs, we assume that all subprocess interactions are independent. In this case, the number of interactions can be described by a Poisson distribution. In our determination of the relative number of correlated and uncorrelated pairs, it is convenient to consider the impactparameter integrated $Q \bar{Q}$ cross section,

$$
\sigma^{p A \rightarrow Q \bar{Q}+X}=\int d^{2} \mathbf{b}\left(1-e^{-T_{A}(\mathbf{b}) \tilde{\sigma}^{Q \bar{Q}}}\right)=\int d^{2} \mathbf{b} \sum_{N=1}^{\infty} \frac{\bar{N}_{Q \bar{Q}}^{N}(\mathbf{b}) e^{-\bar{N}_{Q \bar{Q}}(\mathbf{b})}}{N !},
$$

where $\mathbf{b}$ is the impact parameter. The number of $Q \bar{Q}$ pairs produced as a function of impact parameter is $\bar{N}_{Q \bar{Q}}(\mathbf{b})=T_{A}(\mathbf{b}) \tilde{\sigma}^{Q \bar{Q}}$ with $T_{A}(\mathbf{b})=\int d z \rho_{A}(\mathbf{b}, z)$ the thickness function of a nucleus with density $\rho_{A}(\mathbf{b}, z)$.

Multiplying the right hand side of Eq. (13) by the unit normalized $Q \bar{Q}$ cross section,

$$
1^{N}=\left(\frac{1}{\tilde{\sigma}^{Q \bar{Q}}} \int d^{3} \vec{p}_{Q} d^{3} \vec{p}_{\bar{Q}} \frac{d \tilde{\sigma}^{Q \bar{Q}}}{d^{3} \vec{p}_{Q i} d^{3} \vec{p}_{\bar{Q} i}}\right)^{N},
$$

and differentiating with respect to $d^{3} \vec{p}_{Q} d^{3} \vec{p}_{\bar{Q}}$ we find

$$
\begin{aligned}
\frac{d \sigma^{\mathrm{p} A \rightarrow Q \bar{Q}+X}}{d^{3} \vec{p}_{Q} d^{3} \vec{p}_{\bar{Q}}}= & \int d^{2} \mathbf{b} \sum_{N=1}^{\infty} \frac{\bar{N}_{Q \bar{Q}}^{N}(\mathbf{b}) e^{-\bar{N}} \overline{Q \bar{Q}}^{(\mathbf{b})}}{N !} \prod_{i=1}^{N}\left(\frac{1}{\tilde{\sigma}^{Q \bar{Q}}} \int d^{3} \vec{p}_{Q i} d^{3} \vec{p}_{\bar{Q} i} \frac{d \tilde{\sigma}^{Q \bar{Q}}}{d^{3} \vec{p}_{Q i} d^{3} \vec{p}_{\bar{Q} i}}\right) \\
& \times\left(\sum_{j, k=1}^{N} \delta^{(3)}\left(\vec{p}_{Q}-\vec{p}_{Q j}\right) \delta^{(3)}\left(\vec{p}_{\bar{Q}}-\vec{p}_{\bar{Q} k}\right)\right) .
\end{aligned}
$$

We have written the power $N$ in Eq. (14) as a product in order to separate the momenta $\vec{p}_{i}$ of different subcollisions. We have also written the delta functions as a sum over $j$ and $k$ in order to indicate to which subcollision they belong. When considering correlated pairs, both quarks come from the same subcollision so that $j=k$. In the case of uncorrelated pairs, $j \neq k$, the $Q$ and $\bar{Q}$ are picked from different subcollisions.

Thus for correlated pairs, $j=k$, we have

$$
\begin{aligned}
\frac{d \sigma_{\text {corr }}^{\mathrm{p} A \rightarrow Q \bar{Q}+X}}{d^{3} \vec{p}_{Q} d^{3} \vec{p}_{\bar{Q}}} & =\int d^{2} \mathbf{b} \sum_{N=1}^{\infty} \frac{\bar{N}_{Q \bar{Q}}^{N}(\mathbf{b}) e^{-\bar{N}_{Q \bar{Q}}(\mathbf{b})}}{N !} N \frac{1}{\tilde{\sigma}^{Q \bar{Q}}} \frac{d \tilde{\sigma}^{Q \bar{Q}}}{d^{3} \vec{p}_{Q} d^{3} \vec{p}_{\bar{Q}}} \\
& =\int d^{2} \mathbf{b} T_{A}(\mathbf{b}) \frac{d \tilde{\sigma}^{Q \bar{Q}}}{d^{3} \vec{p}_{Q} d^{3} \vec{p}_{\bar{Q}}} .
\end{aligned}
$$


Similarly, for uncorrelated pairs, $j \neq k$,

$$
\begin{aligned}
\frac{d \sigma_{\text {uncorr }}^{\mathrm{p} A \rightarrow Q \bar{Q}+X}}{d^{3} \vec{p}_{Q} d^{3} \vec{p}_{\bar{Q}}} & =\int d^{2} \mathbf{b} \sum_{N=1}^{\infty} \frac{\bar{N}_{Q \bar{Q}}^{N}(\mathbf{b}) e^{-\bar{N}_{Q \bar{Q}}(\mathbf{b})}}{N !} N(N-1) \frac{1}{\tilde{\sigma}^{Q \bar{Q}}} \frac{d \tilde{\sigma}^{Q \bar{Q}}}{d^{3} \vec{p}_{Q}} \frac{1}{\tilde{\sigma}^{Q \bar{Q}}} \frac{d \tilde{\sigma}^{Q \bar{Q}}}{d^{3} \vec{p}_{\bar{Q}}} \\
& =\int d^{2} \mathbf{b} T_{A}^{2}(\mathbf{b}) \frac{d \tilde{\sigma}^{Q \bar{Q}}}{d^{3} \vec{p}_{Q}} \frac{d \tilde{\sigma}^{Q \bar{Q}}}{d^{3} \vec{p}_{\bar{Q}}} .
\end{aligned}
$$

We use the definition of the nuclear overlap function, $T_{A B}(\mathbf{b})=\int d \mathbf{s} T_{A}(\mathbf{s}) T_{B}(\mathbf{b}-\mathbf{s})$, with $A=B$ to obtain the integral over $\mathbf{b}$ in Eq. (17), $\int d^{2} \mathbf{b} T_{A}^{2}(\mathbf{b})=T_{A A}(\mathbf{0})=$ $A^{2} /\left(\pi R_{A}^{2}\right)$. The last equality is obtained by assuming diffuse Woods-Saxon distributions for the nuclear density. Thus, for correlated pairs we find

$$
\frac{d \sigma_{\text {corr }}^{\mathrm{p} A \rightarrow Q \bar{Q}+X}}{d^{3} \vec{p}_{Q} d^{3} \vec{p}_{\bar{Q}}}=A \frac{d \tilde{\sigma}^{Q \bar{Q}}}{d^{3} \vec{p}_{Q} d^{3} \vec{p}_{\bar{Q}}},
$$

while for uncorrelated pairs

$$
\frac{d \sigma_{\text {uncorr }}^{\mathrm{p} A \rightarrow Q \bar{Q}+X}}{d^{3} \vec{p}_{Q} d^{3} \vec{p}_{\bar{Q}}}=\frac{A^{2}}{\pi R_{A}^{2}} \frac{d \tilde{\sigma}^{Q \bar{Q}}}{d^{3} \vec{p}_{Q}} \frac{d \tilde{\sigma}^{Q \bar{Q}}}{d^{3} \vec{p}_{\bar{Q}}} .
$$

The double differential cross section for the pairs, the right-hand side of Eq. (18), was defined in Eq. (11). The single $Q$ cross section, $d \tilde{\sigma}^{Q \bar{Q}} / d^{3} \vec{p}_{Q}$, is the integral of Eq. (11) over $d^{3} \vec{p}_{\bar{Q}}$. Dividing Eqs. (18) and (19) by $A$ gives the $Q \bar{Q}$ production cross section per nucleon.

The above formalism can be extented to $A B$ collisions by simply replacing the thickness function $T_{A}$ in Eq. (13) by the nuclear overlap function $T_{A B}$.

As seen in Eq. (19), the uncorrelated pair cross section is proportional to the product of two independent single heavy quark cross sections. The heavy quark cross sections are, in turn, proportional to the lepton cross sections. This proportionality to the lepton cross sections could be utilized to study the ratio of cross sections of uncorrelated lepton pairs from $\mathrm{p} A$ and pp collisions. This uncorrelated ratio would probe the square of the nuclear modifications of gluon distribution function, $\left(R_{g}^{A}\right)^{2}$, instead of $R_{g}^{A}$. Because the uncorrelated pair cross section is the product of independent single lepton distributions, lepton pairs produced by leptons from two different nucleon-nucleon collisions can be used to simulate uncorrelated pairs.

\section{Numerical inputs}

We calculate the lepton pair cross section in Eq. (2) by generating lepton pairs in proton-nucleus collisions by Monte Carlo and binning them according to their mass and rapidity. We calculate $\left\langle x_{1}\right\rangle,\left\langle x_{2}\right\rangle$ and $\langle Q\rangle$ in each $\left(y_{\bar{l}}, M_{\bar{l}}\right)$ bin. The $\left\langle x_{2}\right\rangle$ regions 
probed are indicated in Fig. 1. For simplicity, since isospin effects do not play a role in $g g \rightarrow Q \bar{Q}$, we have assumed that the nuclei consist of $A$ protons. We have used the MRST LO (central-gluon) parton distribution functions [18] from PDFLIB [19] along with the EKS98 14 nuclear modifications. The quark masses are $m_{c}=1.2 \mathrm{GeV}$ and $m_{b}=4.75 \mathrm{GeV}$ while the meson masses are $m_{D}=1.8693 \mathrm{GeV}$ and $m_{B}=5.278 \mathrm{GeV}$. In $c \bar{c}$ production we have chosen the factorization scale to be twice the quark transverse mass, $Q^{2}=4 m_{T_{c}}^{2}$, because the lowest values of $m_{T_{c}}^{2}$ might otherwise be below the minimum $Q^{2}$ of the parton distribution set. Since the transverse mass is larger in $b \bar{b}$ production, we use $Q^{2}=m_{T_{b}}^{2}$.

The $c$ quark fragments into $D^{+}, D^{0}$ and $D_{s}$ mesons with respective relative weights 1:1:0.3 [20]. Here we neglect $D_{s}$ mesons and allow $c$ quarks to only fragment to $D^{+}$or to $D^{0}$ with equal probabilities which should not significantly affect the results. Similarly: $\bar{c} \rightarrow D^{-}$and $\bar{D}^{0} ; b \rightarrow B^{+}$and $\bar{B}^{0}$; and $\bar{b} \rightarrow B^{-}$and $B^{0}$.

In order to obtain sufficiently high statistics, we generated $2 \times 10^{6} Q \bar{Q}$ pairs and let each pair fragment $10^{3}$ times. We used JETSET/PYTHIA subroutines [20] for meson decays to leptons. To further improve statistics and reduce the runtime of the calculation, we allowed leptonic decays only. The final normalization is obtained using the leptonic branching ratios. The total $Q \bar{Q}$ production cross sections per nucleon needed for normalization are given in Table 1 .

We also generated uncorrelated lepton pairs from two $Q \bar{Q}$ pairs which then separately fragment and decay. The lepton pairs originating from different quark pairs are the uncorrelated ones. This is the origin of the factor of two in the previous paragraph.

We then binned all correlated and uncorrelated $e^{ \pm} e^{\mp}, e^{ \pm} \mu^{\mp}$, and $\mu^{ \pm} \mu^{\mp}$ pairs. We accept only leptons which originate directly from $D \bar{D}$ and $B \bar{B}$ meson decays and neglect the background from secondary or tertiary leptons from e.g. $D^{+} \rightarrow K^{+} X \rightarrow \mu^{+} X^{\prime}$.

Our pp and $\mathrm{p} A$ results are calculated for $\operatorname{SPS}(\sqrt{s}=17.3 \mathrm{GeV}), \operatorname{RHIC}(\sqrt{s}=200$ $\mathrm{GeV})$ and LHC $(\sqrt{s}=5500 \mathrm{GeV})$, using the same center of mass energies as in $A A$ collisions to probe the same $x$ values in all collisions. At the SPS and LHC, we assume a Pb $(A=208)$ target and an $\mathrm{Au}(A=197)$ target for RHIC. The new NA60 [21] experiment at the SPS makes it possible to detect muon pairs in the range $0 \leq \eta_{\mu} \leq 1$. Since the low energy makes $b \bar{b}$ production negligible, only $c \bar{c}$ production is studied at the SPS. At RHIC, the PHENIX detector [22] is designed to measure $e e, e \mu$ and $\mu \mu$ pairs. The detector has two electron and two muon arms. The acceptance of electron arms is $-0.35<\eta_{e}<0.35$ in pseudorapidity and $\pm\left(22.5^{\circ} \leq \phi_{e} \leq 112.5^{\circ}\right)$ in azimuth. The forward muon arm covers the pseudorapidity region $1.15<\eta_{\mu}<2.44$ with almost full azimuthal acceptance. The backward muon arm has a similar acceptance. We only consider leptons from the forward arm so as not to mix regions of $x_{2}$. The ALICE [23] detector is designed to study nucleus-nucleus interactions at the LHC. They will measure electrons at central rapidity, $\left|\eta_{e}\right| \leq 0.9$, and muons in a forward muon spectrometer with $2.5 \leq \eta_{\mu} \leq 4$. We impose the cuts described above on the lepton pseudorapidity in order to simulate detector acceptances. These are summarized in Table 2]. However, 


\begin{tabular}{cccc}
\hline \hline & SPS & RHIC & LHC \\
\hline electron & $0.0 \leq \eta_{e} \leq 1.0$ & $-0.35 \leq \eta_{e} \leq 0.35$ & $-0.9 \leq \eta_{e} \leq 0.9$ \\
muon & $0.0 \leq \eta_{\mu} \leq 1.0$ & $1.15 \leq \eta_{\mu} \leq 2.44$ & $2.5 \leq \eta_{\mu} \leq 4.0$ \\
\hline \hline
\end{tabular}

Table 2: The cuts on the pseudorapidity of single leptons for the three energy regimes studied are indicated both for electrons and muons.

no cuts on minimum lepton energy or azimuthal angle were imposed.

\section{Results}

We begin by comparing the ratios of lepton pair cross sections with the input $R_{g}^{A}$. Figure 2 shows the ratio of differential cross sections of $e^{ \pm} e^{\mp}$ and $\mu^{ \pm} \mu^{\mp}$ pairs from correlated $D \bar{D}$ and $B \bar{B}$ decays in $\mathrm{p} A$ and pp collisions at the SPS, RHIC and LHC energies as a function of the lepton pair invariant mass (solid curves). These results are compared to the ratios $R_{g}^{A}\left(\left\langle x_{2}\right\rangle,\langle Q\rangle\right)$ (dashed curves) and $R_{g}^{A}\left(\left\langle x_{2}\right\rangle, \sqrt{\left\langle Q^{2}\right\rangle}\right.$ ) (dotteddashed curves) at the average $x_{2}$ and $Q$ of each mass bin summed over $y_{\bar{l}}$. All the results are integrated over the rapidity interval given in Table 2 . For brevity, we define the ratio of the cross sections as a function of lepton pair mass as

$$
r_{\sigma} \equiv \frac{d \sigma^{\mathrm{p} A} / d M}{d \sigma^{\mathrm{pp}} / d M}
$$

Note that $r_{\sigma}$ follows $R_{g}^{A}$ to a very good approximation at all energies. The higher the energy, the better the agreement: at LHC $r_{\sigma}$ agrees with $R_{g}^{A}$ very well. Note also that $R_{g}^{A}\left(\left\langle x_{2}\right\rangle,\langle Q\rangle\right) \approx R_{g}^{A}\left(\left\langle x_{2}\right\rangle, \sqrt{\left\langle Q^{2}\right\rangle}\right)$ in all cases.

The results shown in Fig. 2 are obtained using a delta function for the fragmentation. If the Peterson function is used with $\epsilon_{c}=0.15$ and $\epsilon_{b}=\left(m_{c} / m_{b}\right)^{2} \epsilon_{c}$ |16 , the outcome is very similar. The difference in $r_{\sigma}$ due to the fragmentation function is smaller than or on the order of the statistical fluctuations in the Monte Carlo.

The ratio $r_{\sigma}$ always lies below $R_{g}^{A}$ for two reasons. First, some fraction of $Q \bar{Q}$ production is through the $q \bar{q} \rightarrow Q \bar{Q}$ channel. Quark shadowing effects are also included in the calculation of $r_{\sigma}$. Second, the phase space integration smears the shadowing effect relative to $R_{g}^{A}\left(\left\langle x_{2}\right\rangle,\langle Q\rangle\right)$. The $q \bar{q}$ contribution decreases with energy while $R_{g}^{A}$ changes more slowly over the $x_{2}$ region at the LHC, leading to better agreement at this energy.

We have studied the influence of the $q \bar{q}$ channel by turning off the quark distributions in our simulation, i.e. we fix $f_{q}^{A}=f_{\bar{q}}^{A}=0$ for all quark flavours. The resulting ratio $r_{\sigma}\left(f_{q}^{A}=0\right)$ always lies above $r_{\sigma}$, indicating that quark effects reduce the cross section ratios. However, $r_{\sigma}\left(f_{q}^{A}=0\right)$ is still below $R_{g}^{A}$, suggesting that other effects influ- 


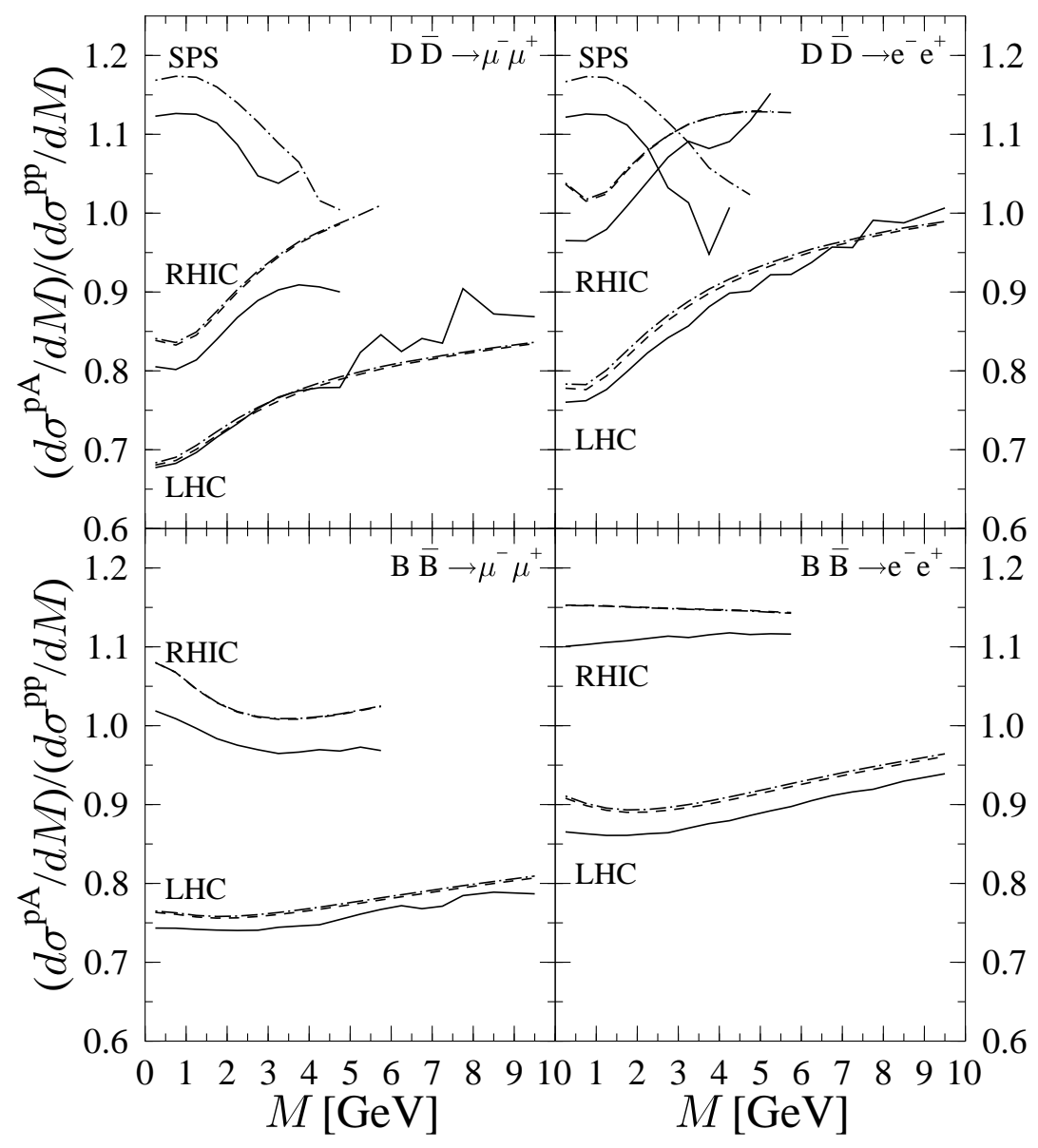

Figure 2: The ratio of $e^{ \pm} e^{\mp}$ and $\mu^{ \pm} \mu^{\mp}$ pair cross sections from correlated $D \bar{D}$ and $B \bar{B}$ decays in $\mathrm{p} A$ and pp collisions, $r_{\sigma}$, defined in Eq. (20), at SPS, RHIC and LHC energies (solid curves). The nuclear gluon distribution, $R_{g}^{A}\left(x_{2}, Q\right)$, at $\left\langle x_{2}\right\rangle$ and $\langle Q\rangle\left(\sqrt{\left\langle Q^{2}\right\rangle}\right)$ of each $M_{\overline{l l}}$ bin is indicated by the dashed (dotted-dashed) curves.

ence $r_{\sigma}$. For example, at the SPS with $M=1.5 \mathrm{GeV}, r_{\sigma} \approx 1.12$ and $r_{\sigma}\left(f_{q}^{A}=0\right) \approx 1.16$ whereas $R_{g}^{A} \approx 1.19$.

In order to estimate the effect of the phase space integration, we reformulate the ratio of lepton pair cross sections as

$$
r_{\sigma}=\frac{\int d(\mathrm{PS})_{Q \bar{Q}} R_{g}^{A}\left(x_{2}, Q\right) h\left(x_{1}, x_{2}, m_{T}, \ldots\right)}{\int d(\mathrm{PS})_{Q \bar{Q}} h\left(x_{1}, x_{2}, m_{T}, \ldots\right)}
$$

where $h\left(x_{1}, x_{2}, m_{T}, \ldots\right)$ is a complicated function which contains the $Q \bar{Q}$ production 
cross section, fragmentation to mesons, and the subsequent meson decay to leptons. The integration over the $Q \bar{Q}$ phase space is denoted by $d(\mathrm{PS})_{Q \bar{Q}}$. We define the average values

$$
\left\langle x_{2}\right\rangle=\int d(\mathrm{PS})_{Q \bar{Q}} x_{2} h\left(x_{1}, x_{2}, m_{T}, \ldots\right), \quad\langle Q\rangle=\int d(\mathrm{PS})_{Q \bar{Q}} Q h\left(x_{1}, x_{2}, m_{T}, \ldots\right),
$$

and expand $R_{g}^{A}$ as a Taylor series around $\left\langle x_{2}\right\rangle$ and $\langle Q\rangle$ up to second order. The terms linear in $\left(x_{2}-\left\langle x_{2}\right\rangle\right)$ and $(Q-\langle Q\rangle)$ are zero after integration and the ratio $r_{\sigma}$ becomes

$$
\begin{aligned}
r_{\sigma}= & R_{g}^{A}\left(\left\langle x_{2}\right\rangle,\langle Q\rangle\right)+\frac{1}{2} \frac{\partial^{2} R_{g}^{A}\left(\left\langle x_{2}\right\rangle,\langle Q\rangle\right)}{\partial x_{2}^{2}}\left\langle\left(x_{2}-\left\langle x_{2}\right\rangle\right)^{2}\right\rangle \\
& +\frac{1}{2} \frac{\partial^{2} R_{g}^{A}\left(\left\langle x_{2}\right\rangle,\langle Q\rangle\right)}{\partial Q^{2}}\left\langle(Q-\langle Q\rangle)^{2}\right\rangle \\
& +\frac{\partial^{2} R_{g}^{A}\left(\left\langle x_{2}\right\rangle,\langle Q\rangle\right)}{\partial x_{2} \partial Q}\left\langle\left(x_{2}-\left\langle x_{2}\right\rangle\right)(Q-\langle Q\rangle)\right\rangle+\ldots .
\end{aligned}
$$

The sum of the correction terms is always negative, thus the ratio $r_{\sigma}$ always lies below $R_{g}^{A}$. A study of the partial derivatives (see Fig. 1) shows that $\partial^{2} R_{g}^{A} / \partial x_{2}^{2}<0$ in the vicinity of $\left\langle x_{2}\right\rangle$ for each energy so that the first correction term is always negative. Near $\langle Q\rangle$, we have $\partial^{2} R_{g}^{A} / \partial Q^{2}>0$ at SPS but $<0$ at RHIC and LHC. The first correction term dominates at SPS but the second one becomes dominant at the LHC energy. The net deviation of $r_{\sigma}$ from $R_{g}^{A}\left(\left\langle x_{2}\right\rangle,\langle Q\rangle\right)$ is then a sum of different terms reflecting both $x$ - and $Q$-dependence of $R_{g}^{A}$. As also seen in Fig. 2, the ratio $r_{\sigma}$ deviates slightly more from $R_{g}^{A}\left(\left\langle x_{2}\right\rangle,\langle Q\rangle\right)$ for the electron pairs than for the muon pairs. The curvature of $R_{g}^{A}$ with $x$ is stronger at larger values of $x$ and due to the differences in rapidity coverage, the average values of $x_{2}$ are larger for electron pairs.

In order to estimate next to leading order (NLO) effects, we have also calculated the differential cross section of $Q \bar{Q}$ production to NLO [24]. The results are shown in Fig. 3 where the ratio $K=\left(d \sigma^{\mathrm{NLO}} / d M\right) /\left(d \sigma^{\mathrm{LO}} / d M\right)$ is given for $\mathrm{p} A$ and pp collisions. This $K$ factor increases with $M$ but decreases with energy. The figure also shows that the $K$ factor for $\mathrm{p} A$ collisions, $K_{A}$, is approximately equal to that for pp collisions, $K_{\mathrm{p}}$, since

$$
\frac{d \sigma_{\mathrm{p} A}^{\mathrm{NLO}}}{d \sigma_{\mathrm{p} A}^{\mathrm{LO}}} \approx \frac{d \sigma_{\mathrm{pp}}^{\mathrm{NLO}}}{d \sigma_{\mathrm{pp}}^{\mathrm{LO}}} \Rightarrow \frac{K_{A} d \sigma_{\mathrm{p} A}^{\mathrm{LO}}}{d \sigma_{\mathrm{p} A}^{\mathrm{LO}}} \approx \frac{K_{\mathrm{p}} d \sigma_{\mathrm{pp}}^{\mathrm{LO}}}{d \sigma_{\mathrm{pp}}^{\mathrm{LO}}} \Rightarrow K_{A} \approx K_{\mathrm{p}} .
$$

This result can be expected even though the $K$ factor is large because the $g g$ channel dominates both LO and NLO $Q \bar{Q}$ production. The $x_{2}$ regions probed at LO and NLO are also very similar. Equation (24) further implies that the ratio of the $\mathrm{p} A$ to pp cross sections at LO and NLO are approximately equal:

$$
\frac{d \sigma_{\mathrm{p} A}^{\mathrm{NLO}}}{d \sigma_{\mathrm{pp}}^{\mathrm{NLO}}} \approx \frac{d \sigma_{\mathrm{p} A}^{\mathrm{LO}}}{d \sigma_{\mathrm{pp}}^{\mathrm{LO}}}
$$




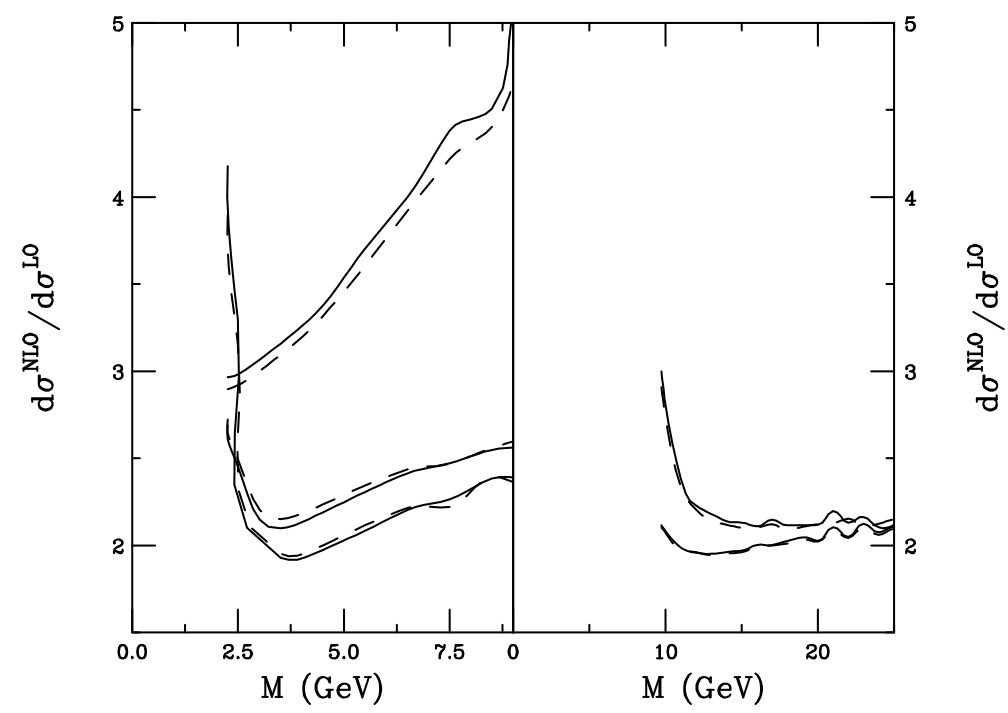

Figure 3: The ratio of the NLO to LO differential cross sections for $Q \bar{Q}$ production as a function of the invariant mass of the $Q \bar{Q}$ pair. Left panel: $c \bar{c}$ pairs produced in $\mathrm{p} A$ (solid) and pp (dashed) collisions at SPS (upper curves), RHIC (middle curves) and LHC (lower curves) energy. Right panel: The same for $b \bar{b}$ production at RHIC (upper curves) and LHC (lower curves).

Thus, the NLO corrections to the ratios in Fig. 2 should be small. A similar result was found for Drell-Yan production at low masses [25, 26.

As explained earlier, the uncorrelated pairs might be used to obtain the ratio $\left(R_{g}^{A}\right)^{2}$. If the nuclear effects turn out to be too small to be measured, that is, if $R_{g}^{A} \sim 1$, the squared ratio might still be observable. We test whether the ratio of uncorrelated lepton pair cross sections, $r_{\sigma}^{\text {uncorr }}$ agrees with $\left(R_{g}^{A}\right)^{2}$ at the LHC where the probability of uncorrelated production is largest. Based on our previous discussion of the $K$ factor, the NLO effects in the ratio $r_{\sigma}^{\text {uncorr }}$ are expected to remain small.

The results for $D \bar{D}$ decays at the LHC are shown in Fig. 1 . It appears that the ratio of cross sections does follow the slope of $\left[R_{g}^{A}(\langle x\rangle,\langle Q\rangle)\right]^{2}$. However, the difference between the ratios is slightly larger than in the correlated case. The origin of this difference can be understood by expanding two $R_{g}^{A}$ factors in the ratio of uncorrelated cross sections, $r_{\sigma}^{\text {uncorr }}$, as in Eq. (23), and noting that the deviations $\left\langle Q^{2}\right\rangle-\langle Q\rangle^{2}$ and $\left\langle x_{2}^{2}\right\rangle-\left\langle x_{2}\right\rangle^{2}$ are larger for uncorrelated pairs. Integration over the rapidity of one of the leptons in a correlated pair increases the allowed phase space and thus the rapidity range of the remaining lepton. This greater available phase space directly increases the variation of $x_{2}$ and $Q$ for uncorrelated pairs, leading to the larger deviations between $r_{\sigma}^{\text {uncorr }}$ and $\left(R_{g}^{A}\right)^{2}$.

The slopes of the ratios $r_{\sigma}$ and $R_{g}^{A}$ (see Fig. 2) are determined by the momentum fractions probed by the interaction, as seen in a comparison with Fig. 11. The average 


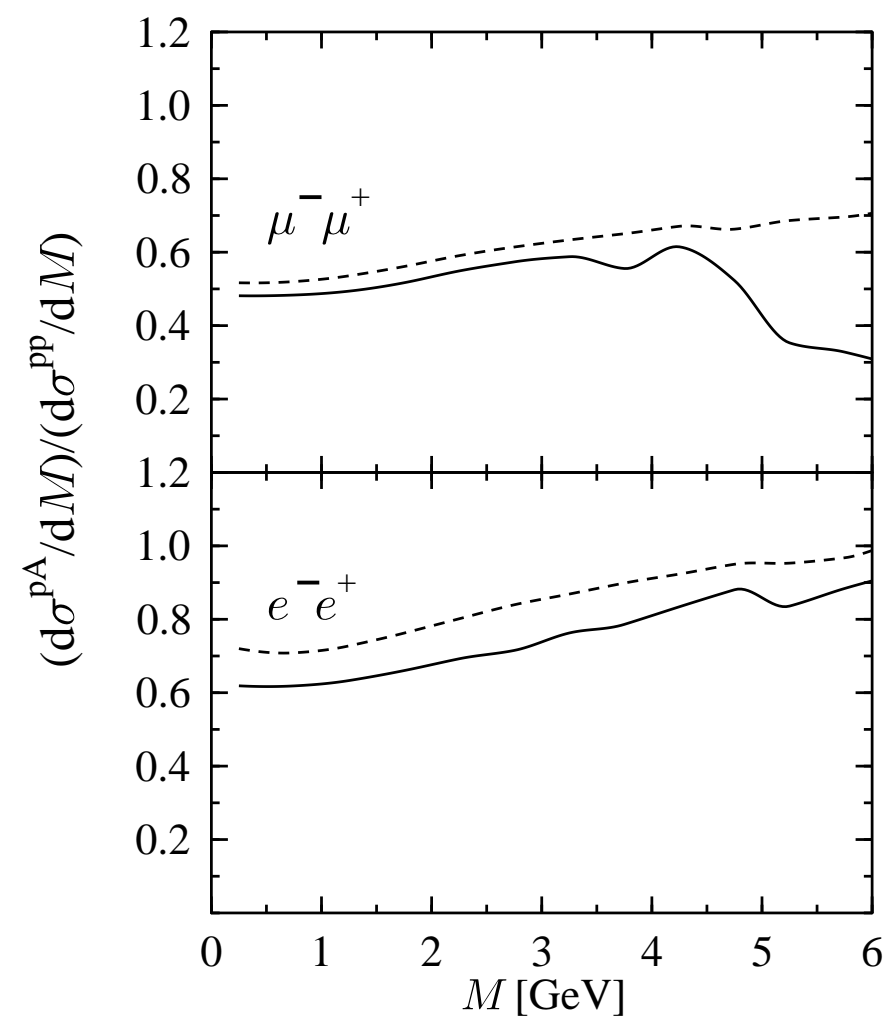

Figure 4: Comparison of $\left[R_{g}^{A}(\langle x\rangle,\langle Q\rangle)\right]^{2}$ (dashed) and uncorrelated lepton pair cross sections, $r_{\sigma}^{\text {uncorr }}$, for $D \bar{D}$ pair decays (solid) at the LHC.

momentum fraction of the parton from nucleus, $\left\langle x_{2}\right\rangle$, with muon pairs is $0.14 \leq\left\langle x_{2}\right\rangle \leq$ 0.32 at the SPS, indicated in Fig. 1. In this $x$ region, the ratio $R_{g}^{A}\left(x, Q^{2}\right)$ is decreasing. At RHIC, the range of momentum fractions probed is $0.003 \leq\left\langle x_{2}\right\rangle \leq 0.012$, where the ratio is increasing quite rapidly. Finally, at the LHC, $3 \times 10^{-5} \leq\left\langle x_{2}\right\rangle \leq 2 \times 10^{-4}$ where $R_{g}^{A}\left(x, Q^{2}\right)$ is almost independent of $x$. The values of $\left\langle x_{2}\right\rangle$ are typically larger for electron pairs at collider energies because the electron coverage is more central than the muon coverage, as seen in Fig. 5 and Table 2 .

We note here that the behaviour of $R_{g}^{A}\left(x, Q^{2}\right)$ depends not only on $x_{2}$ but also on $Q^{2}$. Therefore the slope of $R_{g}^{A}\left(\langle x\rangle,\left\langle Q^{2}\right\rangle\right)$, seen in Fig. 2, does not directly follow any of the curves shown in Fig. 1 but evolves with $Q^{2}$ from one curve to another. The average values $\left\langle Q^{2}\right\rangle$ of $c \bar{c}$ decays are $7.58 \leq\left\langle Q^{2}\right\rangle \leq 48.5 \mathrm{GeV}^{2}$ at the SPS, $9.46 \leq\left\langle Q^{2}\right\rangle \leq 141$ $\mathrm{GeV}^{2}$ at RHIC, and $11.4 \leq\left\langle Q^{2}\right\rangle \leq 577 \mathrm{GeV}^{2}$ at the LHC. The corresponding values of $\langle Q\rangle$ are shown in Fig. 5. The minimum average $Q^{2}$ are larger for $b \bar{b}$ production, $32.0 \leq\left\langle Q^{2}\right\rangle \leq 54.3 \mathrm{GeV}^{2}$ at RHIC and $37.9 \leq\left\langle Q^{2}\right\rangle \leq 156 \mathrm{GeV}^{2}$ at LHC.

The lepton pair cross sections from $B \bar{B}$ decays are also shown in Fig. 2. The results 


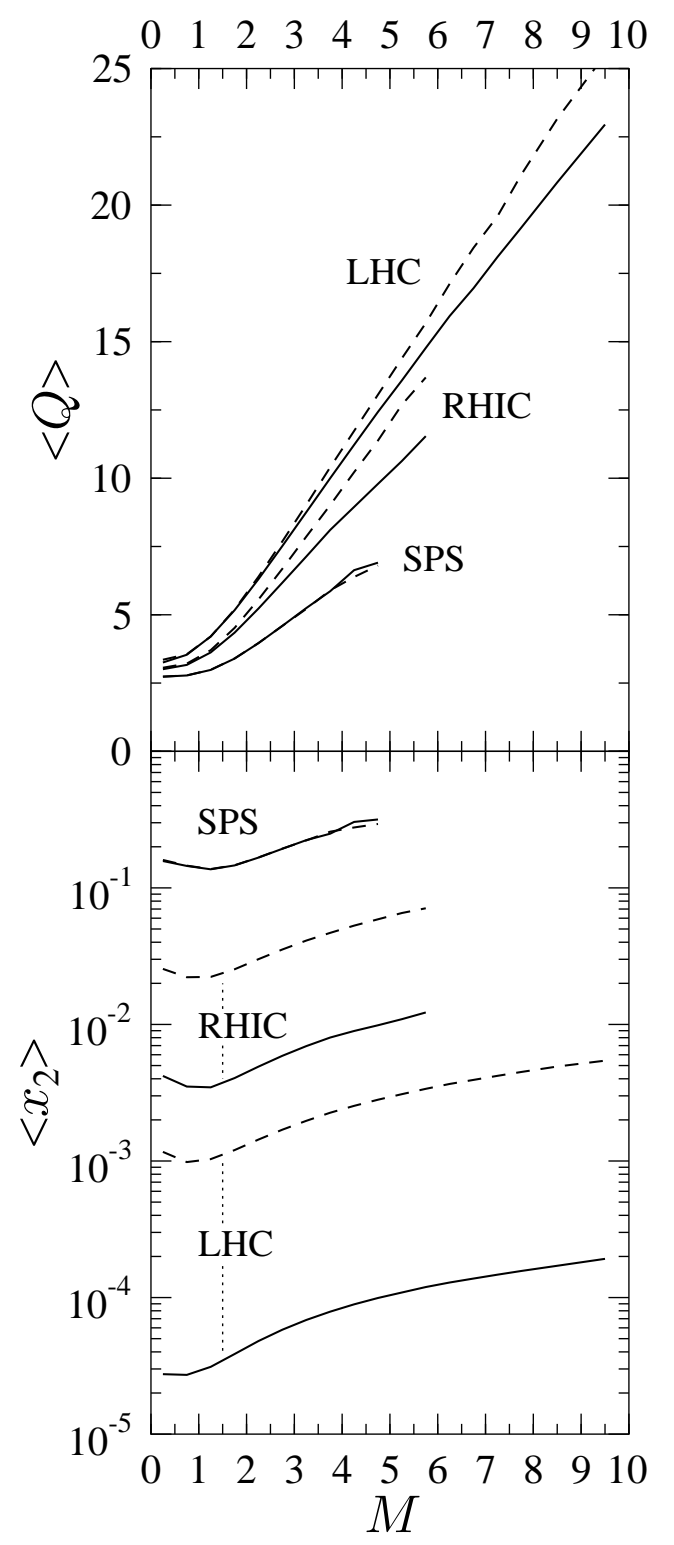

Figure 5: We give $\left\langle x_{2}\right\rangle$ and $\langle Q\rangle$ as a function of pair mass $M$ for $D \bar{D}$ decays at the three energies. The solid lines are for $\mu^{-} \mu^{+}$pairs while the dashed is for $e^{-} e^{+}$pairs.

are quite similar to those from $D \bar{D}$ decays: at RHIC, the difference between $r_{\sigma}$ and $R_{g}^{A}$ is $\sim 5 \%$ but decreases to $1-2 \%$ at the LHC. The slopes of these curves are different than for $D \bar{D}$, they are almost constant with $M$. This constancy is due to the much narrower $x$ and higher $Q^{2}$ regions probed by $b \bar{b}$ production. In a narrow $x$ region, the change in slope with $x$ is small while at high $Q^{2}$, evolution is slower, as seen in Fig. 11. 

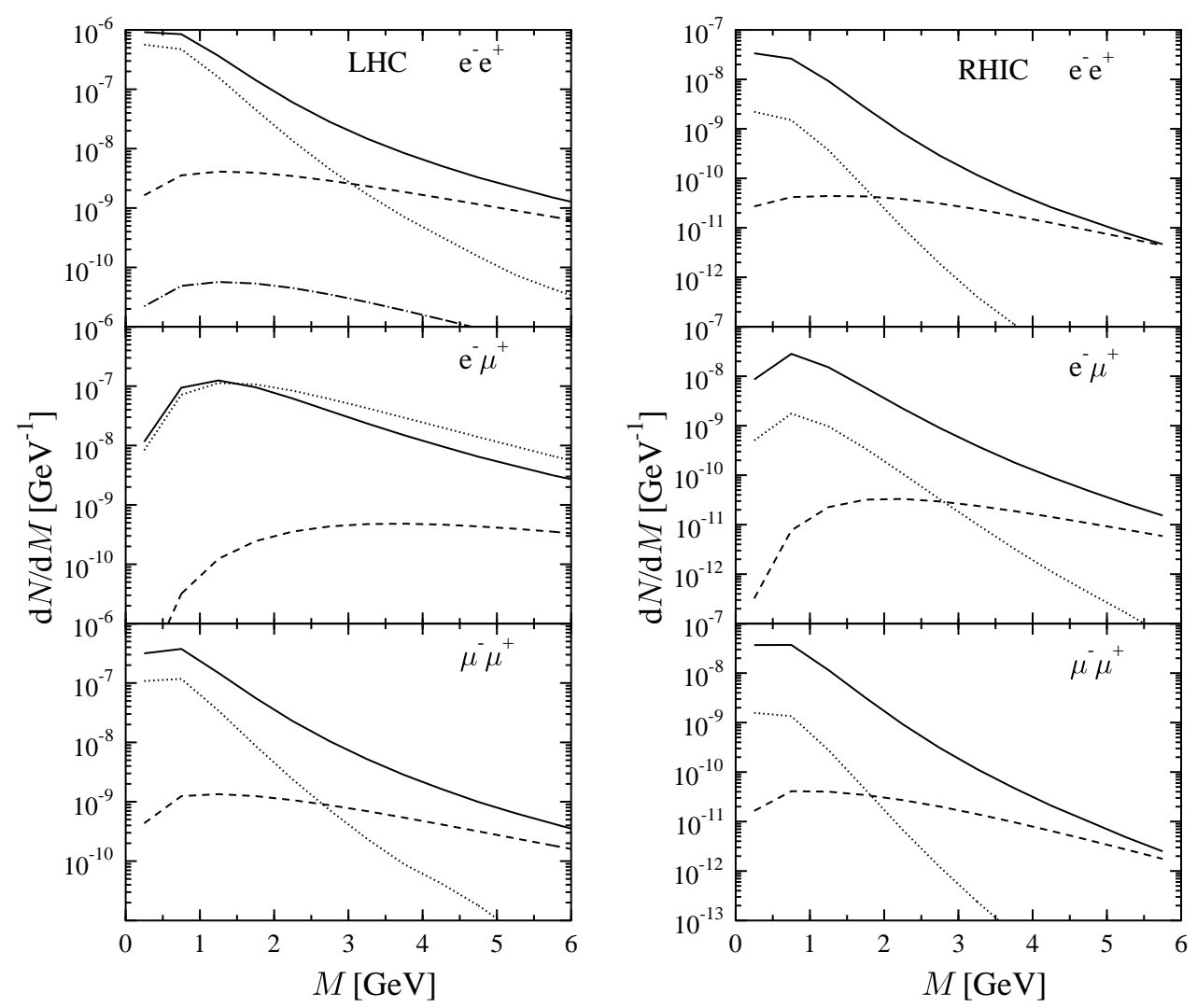

Figure 6: Comparison of lepton pairs from correlated and uncorrelated $D \bar{D}$ and $B \bar{B}$ pairs at LHC (left) and RHIC (right). The differential number densities of $e^{ \pm} e^{\mp}, e^{ \pm} \mu^{\mp}$ and $\mu^{ \pm} \mu^{\mp}$ pairs are given as a function of $M$. The lepton pairs originate from correlated $D \bar{D}$ (solid), correlated $B \bar{B}$ (dashed), uncorrelated $D \bar{D}$ (dotted) and uncorrelated $B \bar{B}$ (dot-dashed) decays. The results are shown without $K$ factors.

Figure 6 shows the mass dependence of the differential number density distribution of lepton pairs in central collisions, obtained from Eqs. (16) and (17) through differentiation with respect to $\mathbf{b}$ and setting $\mathbf{b}=0$. These results are shown without any $K$ factors, so that the final result will be shifted when a $K$ factor is applied. In particular, the relative normalization of the curves will change since the correlated pairs will be multiplied by $K$ while the uncorrelated pairs are increased by $K^{2}$. Taking this correction into account, one sees that at the LHC the number of uncorrelated pairs at $M \gtrsim 2-3 \mathrm{GeV}$ is of the same order as the correlated ones. At higher $M$, the uncorrelated pairs are reduced and can be safely neglected, except in the $e \mu$ configuration where the uncorrelated pairs are larger. On the other hand, lepton pairs originating from $B \bar{B}$ decays are negligible at low $M$ but become important at $M \gtrsim 5-6 \mathrm{GeV}$. At RHIC, leptons from correlated $D \bar{D}$ decays clearly dominate those from uncorrelated 
pairs over all masses so that the uncorrelated pairs can be neglected. Note that at this energy also, leptons from $B \bar{B}$ decays are of the same magnitude as the correlated $D \bar{D}$ decays for $M \gtrsim 4-5 \mathrm{GeV}$.

In Ref. 14 it was shown that, in $A A$ collisions, lepton pairs from uncorrelated heavy quark decays tend to have higher masses than those from correlated decays. This is because the rapidity gap, $\Delta y$, between the quarks is larger when they are uncorrelated and $M^{2} \propto 1+\cosh \Delta y$. The uncorrelated rate is much smaller in $\mathrm{p} A$ interactions, however, which would reduce the probability of a large $\Delta y$ relative to $A A$ collisions. Including a finite rapidity cut severely reduces the number of leptons from uncorrelated decays falling within the acceptance. The effect of the rapidity cut is to reduce the average $M$ for uncorrelated relative to correlated pairs. Thus in Fig. 6 lepton pairs from uncorrelated decays are most strongly reduced at large masses when the rapidity acceptance is small, as in the $e^{+} e^{-}$coverage at RHIC. Since the $e \mu$ measurement involves mid-rapidity electrons and forward (or backward) muons, the rapidity coverage is largest and fewer uncorrelated decays are rejected. In fact, the larger $e \mu$ coverage at the LHC as well as the higher $D \bar{D}$ rates lead to more uncorrelated pairs at larger masses than correlated pairs.

The results in Fig. 6 are for opposite-sign lepton pairs only. The uncorrelated yield can be determined using a like-sign subtraction since like-sign pairs are all trivially uncorrelated. The number of uncorrelated opposite-sign pairs is equivalent to the number of like-sign pairs so it is relatively easy to estimate the real amount of uncorrelated pairs. After this estimate, the uncorrelated pairs can be used to study $\left(R_{g}^{A}\right)^{2}$, as shown in Fig. 4 .

Finally we wish to mention that along with "normal" uncorrelated lepton pairs originating from $D \bar{D}$ and $B \bar{B}$ decays a number of "crossed events", i.e. uncorrelated leptons from $D \bar{B}$ and $B \bar{D}$ decays, are also produced. Their rate lies between those from uncorrelated $D \bar{D}$ and $B \bar{B}$ pairs. The total number of observed lepton pairs from heavy quark decays is the sum of all pairs from $D \bar{D}, B \bar{B}, D \bar{B}$ and $\bar{D} B$ decays. It is necessary to know the relative number of pairs produced in each case in order to decompose the results, as in Fig. 6. However, since uncorrelated $B \bar{B}$ pairs are generally negligible, we can also neglect these "crossed events" and assume that it is possible to deduce which lepton pairs came from correlated $D \bar{D}$ pairs. We do not consider any "crossed events" from $D$ or $B$ decays combined with leptons from $\pi$ or $K$ decays. These should predominantly appear at lower masses and can be removed by like-sign subtraction.

\section{Conclusions}

We have calculated lepton pair production from heavy quark decays in $\mathrm{p} A$ and pp collisions using the EKS98 nuclear modifications to the parton distributions. We have shown that the resulting cross section ratios of $\mathrm{p} A$ to $\mathrm{pp}$ rates reflect the initial nu- 
clear modifications of gluons rather well, as seen in Fig. 2. The deviations caused by phase space and the finite quark contributions are analyzed. Based on these results, we conclude that if the ratio of leptonic cross sections were measured within an acceptable accuracy, the nuclear gluon distributions could be determined. Several such measurements have been proposed.

The NA60 experiment [21] at the SPS plans to measure charm production through lepton decays. At this energy, a $20 \%$ antishadowing in $R_{g}^{A}$ manifests itself as a $10 \%$ enhancement in the ratio of cross sections due to the finite quark component. Assuming the measurements could be done to $\sim 10 \%$ accuracy, NA60 results could be used to pinpoint the behaviour of nuclear gluon distribution in the range $0.17 \lesssim x \lesssim 0.4$.

At higher energies, the $g g$ channel becomes more important so that $R_{g}^{A}$ reflects the cross section ratio more closely. At RHIC, the PHENIX detector [22] will use lepton pairs to measure heavy quark production at $x$ values as low as $3 \times 10^{-3}$. In this $x$ region, the $Q^{2}$ dependence of gluon modifications should be larger than at the SPS. The nuclear effects vary from $20 \%$ shadowing for charm to slight antishadowing for bottom. The increased importance of the gluon channel reduces the difference between $d \sigma_{\mathrm{p} A} / d \sigma_{\mathrm{pp}}$ and $R_{g}^{A}$ to $\sim 5 \%$.

The ALICE detector [23] is designed to study nucleus-nucleus interactions at the LHC. The high LHC energy makes it ideal for probing nuclear effects of gluon distributions and studying saturation effects at low $x$. For $x$ values as low as $3 \times 10^{-5}$, the evolution in $Q^{2}$ should be measurable, as shown in Fig. 1. Here, the nuclear modifications are an $\sim 30 \%$ effect, and the difference between $d \sigma_{\mathrm{p} A} / d \sigma_{\mathrm{pp}}$ and $R_{g}^{A}$ is of the order of one percent.

All three experiments, taken together, provide a large lever arm in $\left\langle x_{2}\right\rangle$ and $\left\langle Q^{2}\right\rangle$ over which to study the nuclear gluon distribution. Measurements at the same energy in $\mathrm{p} A$ and pp interactions with high enough statistics should reveal much new information on $R_{g}^{A}$. A better determination of $R_{g}^{A}$ in turn provides valuable input to the study of $A A$ interactions.

Acknowledgements: We thank P.V. Ruuskanen and Z. Lin for useful discussions. R.V. thanks the Niels Bohr Institute for hospitality during the completion of this work. The work of K.J.E. and V.K. was supported by the Academy of Finland. The work of R.V. was supported in part by the Director, Office of Energy Research, Division of Nuclear Physics of the Office of High Energy and Nuclear Physics of the U. S. Department of Energy under Contract Number DE-AC03-76SF00098.

\section{References}

[1] M. Luo, J. Qiu and G. Sterman, Phys. Rev. D49 (1994) 4493.

[2] R.J. Fries, A. Schäfer, E. Stein and B. Müller, Nucl. Phys. B582 (2000) 537. 
[3] K.J. Eskola, V.J. Kolhinen and P.V. Ruuskanen, Nucl. Phys. B535 (1998) 351.

[4] K.J. Eskola, V.J. Kolhinen and C.A. Salgado, Eur. Phys. J. C9 (1999) 61.

[5] M. Arneodo, Phys. Rep. 240 (1994) 301.

[6] Yu. Dokshitzer, Sov Phys. JETP 46 (1977) 641; V.N. Gribov and L.N. Lipatov, Sov. Nucl. Phys. 15 (1972) 438, 675; G. Altarelli, G. Parisi, Nucl. Phys. B126 (1977) 298.

[7] K.J. Eskola, Nucl. Phys. B400 (1993) 240.

[8] L.V. Gribov, E.M. Levin and M.G. Ryskin, Nucl. Phys. B188 (1981) 555; Phys. Rep. 100 (1983) 1.

[9] A.H. Mueller and J. Qiu, Nucl. Phys. B268 (1986) 427.

[10] D.M. Alde et al. (E772 Collab.), Phys. Rev. Lett. 64 (1990) 2479.

[11] R.E. Tribble et al. (E866/NuSea Collab.), Nucl. Phys. A663 (2000) 761.

[12] M. Arneodo et al. (NM Collab.), Nucl. Phys. B481 (1996) 23.

[13] T. Gousset and H.J. Pirner, Phys. Lett. B375 (1996) 349.

[14] S. Gavin, P.L. McGaughey, P.V. Ruuskanen and R. Vogt, Phys. Rev. C54 (1996) 2606.

[15] Z. Lin and M. Gyulassy, Nucl. Phys. A610 (1996) 476.

[16] C. Peterson, D. Schlatter, I. Schmitt, and P.M. Zerwas, Phys. Rev. D27 (1983) 105.

[17] B.L. Combridge, Nucl. Phys. B151 (1979) 429.

[18] A.D. Martin, R.G. Roberts, W.J. Stirling and R.S. Thorne, Eur. Phys. J. C4 (1998) 463; Phys. Lett. B443, 301 (1998).

[19] H. Plothow-Besch, Comp. Phys. Comm. 75 (1993) 396; Int. J. Mod. Phys. A10 (1995) 2901.

[20] T. Sjöstrand, Comp. Phys. Comm. 82 (1994) 74.

[21] A. Baldit et al. (NA60 Collab.), Proposal SPSC/P316, March 2000; http://na60.web.cern.ch/NA60.

[22] D.P. Morrison et al. (PHENIX Collab.), Nucl. Phys. A638 (1998) 565c. 
[23] ALICE Collaboration, Technical Proposal, CERN/LHCC 95-71, ALICE Collaboration, Addendum to the Letter of Intent, CERN/LHCC 95-24, ALICE Collaboration, Addendum to ALICE Proposal, CERN/LHCC 99-13.

[24] R.V. Gavai et al., Int. J. Mod. Phys. A10 (1995) 3043.

[25] V. Emel'yanov, A. Khodinov, S.R. Klein and R. Vogt, Phys. Rev. C61 (2000) 044904.

[26] K.J. Eskola, V.J. Kolhinen, C.A. Salgado and R.L. Thews, JYFL-4/00, LPT Orsay 00-73, hep-ph/0009251, submitted to Eur. Phys. J. C. 